\title{
Dynamic Stochastic Multiattribute Decision-Making That Considers Stochastic Variable Variance Characteristics under Time-Sequence Contingency Environments
}

\author{
Zao-li Yang and Lu-cheng Huang \\ College of Economics and Management, Beijing University of Technology, Beijing 100124, China \\ Correspondence should be addressed to Zao-li Yang; yangzaoli@bjut.edu.cn
}

Received 19 October 2016; Revised 21 January 2017; Accepted 6 February 2017; Published 26 February 2017

Academic Editor: Peide Liu

Copyright @ 2017 Zao-li Yang and Lu-cheng Huang. This is an open access article distributed under the Creative Commons Attribution License, which permits unrestricted use, distribution, and reproduction in any medium, provided the original work is properly cited.

\begin{abstract}
This paper presents a dynamic stochastic decision-making method that considers the characteristics of stochastic variable variances under time-sequence contingency environments for solving stochastic decision-making problems with information from different periods and of indeterminate attribute weights. First, time-sequence weights are obtained using the technique for order preference by similarity to ideal solution (TOPSIS), corresponding with the idea of "stressing the present rather than the past." After determining the time degree and fully considering the characteristics of normally distributed stochastic variable variances, the attribute weight is determined based on vertical projection distance. Decision-making information is then assembled from two dimensions of time-sequence and attributes, based on the two categories of weighted arithmetic averaging operators of normally distributed stochastic variables, resulting in comprehensive dynamic decision-making from single solution dimensions and a priority sequence of solutions per the order relation criteria of normally distributed stochastic variables. Finally, the validity and practicability of the methods proposed in this paper are verified using an example numerical analysis.
\end{abstract}

\section{Introduction}

In modern socioeconomic decision-making systems, people often face decision-making problems with a variety of information conditions, such as stochastic information [1-12], 2-dimension uncertain linguistic information [13, 14], intuitionistic fuzzy information [15-19], intuitionistic trapezoidal fuzzy information [20,21], triangular fuzzy [22], single-valued neutrosophic numbers [23], trapezoidal fuzzy numbers [24], and neutrosophic hesitant fuzzy information [25]. One of them, which have different time-sequence phases and multiple attribute indexes of normally distributed stochastic variables, like street traffic flow, shopping centre popularity at different times, or the customer waiting times, is referred to as dynamic stochastic multiattribute decisionmaking problems.

Compared with dynamic stochastic decision-making, which comprehensively considers multiple time-sequences, stochastic multi-attribute decision-making under a single static time-sequence environment has received more widespread attention from scholars. These research findings mainly expand on information aggregation operators $[1]$, stochastic dominance $[2,3]$, stochastic multiattribute analysis [4-6], set pair connection number analysis [7], prospect stochastic dominance $[8,9]$, bivariate expectation in decision-making [10], probability weighted means [3], and possibility degree interval-valued numbers [11, 12], of attribute weights in an unknown state-further expanding the research boundary of stochastic multiattribute decisionmaking. It is difficult for most decision-making results to be comprehensive and rationally optimised based on single time-sequence nodes. Therefore, past and current decision-making information should also be considered when conducting stochastic decision-making. Appropriately determining the weight of different time-sequences is key to successful dynamic stochastic decision-making. Current 
scholars have mainly developed the following objective methods based on sample information to establish time-sequence weights: discrete time-sequences [12], normal distribution [15], time-sequence information entropy [16], difference geometric progression [26], and exponential distribution [27]. Moreover, scholars such as Park et al. [17], Li et al. [24], Cao et al. [28], and Liu et al. [29] et al. have conducted weight allocation on different time-sequences by relying on expert experience and subjective preference. These methods provide a reference for solving for time-sequence weights in dynamic stochastic problems, but they derive weights based on an agent's preference in each period, which inevitably results in randomness in the time-sequence weight distribution. For instance, methods based on objective information often tend to emphasise time-sequences with more information in an historical period; at these times, sequence weight increases $[12,15,16]$. However, methods that merely rely on the subjective perception of information at different times, owing to diversity in subjective perception, greatly impact the stability of decision-making results [17, 24, 28, 29]. In real life, people pay more attention to the most current information when making judgments of the present. Therefore, this paper focuses on allocating time-sequence weights in accordance with the closeness of historical information to the latest information.

Dynamic stochastic multiattribute decision-making problems possess a time dimension and an attribute dimension; therefore, determining attribute weights is a prerequisite for assembling the attribute information required for the final decision-making result. Relevant scholars have developed a variety of methods for successfully determining attribute weight. For instance, Chen and Li have obtained attribute weights [22] by solving the grey relation function of attribute information per the grey correlation model. Scholars, such as Yue [18], Cao et al. [19], and Zhang et al. [30] et al., have designed linear optimisation models of attribute weight using TOPSIS-based Euclidean distance. $\mathrm{Xu}$ and Wan [31] have calculated attribute weights based on an uncertaintyordered weighted averaging operator and an attribute information ordering method that relates to attribute weight size and attribute evaluation value size and order. This idea provides a basis for attribute weight calculation in normally distributed stochastic decision-making problems; however, due to its assumed conditions and the characteristic requirements of normally distributed stochastic variables, this method's applications are limited. For instance, grey system theory and TOPSIS are based on Euclidean distance. Since Euclidean distance does not consider the distance relationship between a decision-making solution and the positive and negative ideal solutions, the weight calculation can easily lead to a "reversed order" phenomenon, in which the attribute information ordering method assigns weights per the attribute information size criteria, but attribute information is the decision-making information after inverting the normally distributed stochastic variables into common interval numbers, and thus the attribute information of the original normally distributed stochastic variables is lost completely. Therefore, attribute weight information that considers normally distributed stochastic variable characteristics is expanded upon in this paper.

Based on these analyses, this study proposes a dynamic stochastic multiattribute decision-making method that considers random variable variance characteristics in timesequence contingencies with respect to time-sequence and attribute. The remainder of this paper is organised as follows: Section 2 reviews random decision-making and related algorithms in which attribute values are normally distributed stochastic variables. Section 3 provides a time-sequence weight calculation model using the ideal time-sequence solution by introducing the concept of time degree. The validity and applicability of the proposed dynamic stochastic decision-making method are examined in Section 4 using a numerical example. Conclusions and directions for future work are discussed in Section 5.

\section{Background}

Here, we offer a concise overview of the essentials of normally distributed numbers and stochastic variables and the operation rules thereof.

Definition 1 (see [32]). A stochastic (random) variable, usually written as $X$, is a variable whose possible values are numerical outcomes of a random phenomenon. There are two types of stochastic variables: discrete and continuous.

Definition 2 (see [12]). Suppose that the probability density of a continuous type of stochastic variable $X$ is $f(x)=$ $(1 / \sqrt{2 \pi} \sigma) e^{-(x-\mu)^{2} / 2 \sigma^{2}}$, where parameters $\mu$ and $\sigma^{2}$ are the expectation and variance of the stochastic variables, respectively. $X$ is a normal distribution, denoted by $X \sim$ $N\left(\mu, \sigma^{2}\right)$, with a cumulative probability function of $F(x)=$ $(1 / \sqrt{2 \pi} \sigma) \int_{-\infty}^{x} e^{-(t-\mu)^{2} / 2 \sigma^{2}} d t .\{\mu, \sigma\}$ is the normal distribution numbers of stochastic variable $X$, denoted by $x=\{\mu, \sigma\}$, and $\Theta$ is the set of all normal distribution numbers.

Lahdelma et al. (2006) [33] proposed that the stochastic parameters $\sigma$ and $\mu$ depend on the basic discrete sample $\left\{Z^{l}, l=1,2, \ldots, L\right\}$, where $Z$ is criteria values and $l$ is sample size. The stochastic parameter $\mu$ is estimated by the sample mean

$$
\mu \cong \bar{Z}=\frac{\sum_{l=1}^{L} Z^{l}}{L}
$$

and the stochastic parameter $\sigma$ is the square root of the sample variance

$$
\sigma \cong \sqrt{\frac{\sum_{l=1}^{L}\left(Z^{l}-\bar{Z}\right)^{2}}{(L-1)}} .
$$

Definition 3 (see [11]). For two arbitrary normal distribution numbers $x_{1}=\left\{\mu_{1}, \sigma_{1}\right\}$ and $x_{2}=\left\{\mu_{2}, \sigma_{2}\right\}$,

(1) $x_{1} \oplus x_{2}=\left\{\mu_{1}+\mu_{2}, \sqrt{\sigma_{1}^{2}+\sigma_{2}^{2}}\right\}$;

(2) $\lambda x_{1}=\left\{\lambda \mu_{1}, \lambda \sigma_{1}\right\}$;

(3) $\lambda\left(x_{1} \oplus x_{2}\right)=\lambda x_{1} \oplus \lambda x_{2}$. 
Definition 4 (see $[11,12]$ ). For an arbitrary normal distribution number set $x_{j}=\left\{\mu_{j}, \sigma_{j}\right\}$, a normally distributed number weighted arithmetic average (NDNWAA) operator of dimension $n$ is a mapping NDNWAA: $\Theta^{n} \rightarrow \Theta$,

$$
\begin{aligned}
\operatorname{NDNWAA}_{w}\left(x_{1}, x_{2}, \ldots, x_{n}\right) \\
=w_{1} x_{1} \oplus w_{2} x_{2} \oplus \cdots \oplus w_{n} x_{n} \\
=\left\{\sum_{j=1}^{n} w_{j} \mu_{j}, \sqrt{\sum_{j=1}^{n} w_{j}^{2} \sigma_{j}^{2}}\right\},
\end{aligned}
$$

where $w=\left(w_{1}, w_{2}, \ldots, w_{n}\right)^{T}$ is the weight vector of the stochastic variable $X_{j}(j=1,2, \ldots, n)$ attribute, $w_{j} \in[0,1]$, $\sum w_{j}=1$.

Definition 5 (see [12]). Suppose that $x(t)=\left(x\left(t_{1}\right), x\left(t_{2}\right), \ldots\right.$, $\left.x\left(t_{p}\right)\right)$ is a group of normally distributed numbers under $p$ different moments $t_{k}(k=1,2, \ldots, p)$; a dynamic normal distribution number weighted arithmetic average (DNDNWAA) operator of dimension $p$ is a mapping NDNWAA: $\Theta^{p} \rightarrow \Theta$

$$
\begin{aligned}
& \text { DNDNWAA }_{w(t)}\left(x\left(t_{1}\right), x\left(t_{2}\right), \ldots, x\left(t_{p}\right)\right) \\
& =w\left(t_{1}\right) x\left(t_{1}\right) \oplus w\left(t_{2}\right) x\left(t_{2}\right) \oplus \cdots \oplus w\left(t_{p}\right) x\left(t_{p}\right) \\
& =\left\{\sum_{k=1}^{p} w\left(t_{k}\right) \mu\left(t_{k}\right), \sqrt{\sum_{k=1}^{p} w\left(t_{k}\right)^{2} \sigma\left(t_{k}\right)^{2}}\right\},
\end{aligned}
$$

where $w(t)=\left(w\left(t_{1}\right), w\left(t_{2}\right), \ldots, w\left(t_{p}\right)\right)^{T}$ is the weight of timesequence $t_{k}(k=1,2, \ldots, p), w\left(t_{k}\right) \in[0,1], \sum_{k=1}^{p} w\left(t_{k}\right)=1$.

\section{The Dynamic Stochastic Decision-Making Method}

3.1. Problem Description. Suppose that there exists a dynamic stochastic decision-making problem, for which $S=$ $\left\{S_{1}, S_{2}, \ldots, S_{m}\right\}$ is a discrete and independent alternative solution set, $C=\left\{C_{1}, C_{2}, \ldots, C_{n}\right\}$ is the attribute set to which the solution is subject, $w=\left(w_{1}, w_{2}, \ldots, w_{n}\right)^{T}$ is the weight vector, and $w(t)=\left(w\left(t_{1}\right), w\left(t_{2}\right), \ldots, w\left(t_{p}\right)\right)^{T}$ is the time-weight vector. The value of attribute $C_{j}$, to which solution $S_{i}$ is subject at moment $t_{k}$, is denoted as $X_{i j}\left(t_{k}\right)$, which is subject to a normal distribution, denoted by $X_{i j}\left(t_{k}\right) \sim N\left(\mu_{i j}\left(t_{k}\right), \sigma_{i j}\left(t_{k}\right)^{2}\right)$ and its corresponding normal distribution number $x_{i j}\left(t_{k}\right)=$ $N\left(\mu_{i j}\left(t_{k}\right), \sigma_{i j}\left(t_{k}\right)\right)$, forming a primitive decision-making matrix $D_{X\left(t_{k}\right)}=N\left(\mu_{i j}\left(t_{k}\right), \sigma_{i j}\left(t_{k}\right)^{2}\right)_{m \times n}$ based on $p$ moments of decision-making information. Dynamic stochastic decision-making problems consist of multiple dimensions, such as solution, attribute, and time. Determining attribute and time-sequence weights is key to reducing dimensionality and solving dynamic stochastic problems.

3.2. Determination of Time-Sequence Weight. In dynamic stochastic multiattribute decision-making problems, timesequence weight vector $w\left(t_{k}\right)=\left(w\left(t_{1}\right), w\left(t_{2}\right), \ldots, w\left(t_{p}\right)\right)^{T}$ is a prerequisite for assembling time-sequence information and obtaining a decision-making result. Contrary to previous similar research, for example, Sun and $\mathrm{Xu}$ [12] and Tan and Chen [16], which are merely based on the absolute quantity of time-sequence information or completely rely on subjective arbitrary judgments, this paper obtains a timesequence weight vector using TOPSIS and by introducing time-degree criteria in accord with the findings of Yager [34] and Zhang and Zhu [35].

Definition 6 (see [35]). Denote $\lambda=\sum_{k=1}^{p}((p-k) /(p-1)) w\left(t_{k}\right)$, where $0 \leq \lambda \leq 1$ and $\lambda$ is the time degree of the time-sequence weight vector $w\left(t_{k}\right)=\left(w\left(t_{1}\right), w\left(t_{2}\right), \ldots, w\left(t_{p}\right)\right)^{T}$. Time degree describes the degree of attention the decision maker attaches to decision-making information from different timesequence phases. When $\lambda$ decreases, the decision maker attaches more importance to recent information; when $\lambda$ increases, the decision maker attaches more importance to older information.

Based on this analysis and guided by the principle of "stress the present rather than the past," when $\lambda=0$, then $w(t)=(0,0, \ldots, 1)^{T}$, indicating that the decision maker attaches full importance to current information, and $w(t)^{+}=$ $(0,0, \ldots, 1)^{T}$ can be denoted by the positive ideal time-weight vector; when $\lambda=1$, then $w(t)=(1,0, \ldots, 0)^{T}$, indicating that the decision maker attaches full importance to past information, and $w(t)^{-}=(1,0, \ldots, 0)^{T}$ can be denoted by the negative ideal time-weight vector.

Per the definition of Euclidean distance, the distance between time-weight vectors $w^{1}\left(t_{k}\right)=\left(w^{1}\left(t_{1}\right), w^{1}\left(t_{2}\right), \ldots\right.$, $\left.w^{1}\left(t_{p}\right)\right)^{T}$ and $w^{2}\left(t_{k}\right)=\left(w^{2}\left(t_{1}\right), w^{2}\left(t_{2}\right), \ldots, w^{2}\left(t_{p}\right)\right)^{T}$ can be denoted by

$$
d\left(w^{1}\left(t_{k}\right), w^{2}\left(t_{k}\right)\right)=\sqrt{\sum_{k=1}^{p}\left|w^{1}\left(t_{k}\right)-w^{2}\left(t_{k}\right)\right|^{2}} .
$$

Thus, the distances of a time-weight vector $w\left(t_{k}\right)=$ $\left(w\left(t_{1}\right), w\left(t_{2}\right), \ldots, w\left(t_{p}\right)\right)^{T}$ from the positive and negative ideal time-weight vectors are, respectively,

$$
\begin{aligned}
& d\left(w\left(t_{k}\right), w\left(t_{k}\right)^{+}\right)=\sqrt{\sum_{k=1}^{p-1} w\left(t_{k}\right)^{2}+\left(1-w\left(t_{p}\right)\right)^{2}} \\
& d\left(w\left(t_{k}\right), w\left(t_{k}\right)^{-}\right)=\sqrt{\left(1-w\left(t_{1}\right)\right)^{2}+\sum_{k=2}^{p} w\left(t_{k}\right)^{2}}
\end{aligned}
$$

The closeness of time-weight vector $w\left(t_{k}\right)$ to the ideal time-weight vector can be obtained by

$$
C=\frac{d\left(w\left(t_{k}\right), w\left(t_{k}\right)^{-}\right)}{d\left(w\left(t_{k}\right), w\left(t_{k}\right)^{+}\right)+d\left(w\left(t_{k}\right), w\left(t_{k}\right)^{-}\right)} .
$$

The greater the closeness degree $C$ is, the more the attention is attached to current information. To maximise the closeness of a time-sequence weight vector, the following model is optimised based on nonlinear programming under the given time-degree conditions 


$$
\begin{array}{ll}
\max \quad C=\frac{\sqrt{\left(1-w\left(t_{1}\right)\right)^{2}+\sum_{k=2}^{p} w\left(t_{k}\right)^{2}}}{\sqrt{\sum_{k=1}^{p-1} w\left(t_{k}\right)^{2}+\left(1-w\left(t_{p}\right)\right)^{2}}+\sqrt{\left(1-w\left(t_{1}\right)\right)^{2}+\sum_{k=2}^{p} w\left(t_{k}\right)^{2}}}, \\
\text { s.t. } \quad \lambda=\sum_{k=1}^{p} \frac{p-k}{p-1} w\left(t_{k}\right), \\
\quad \sum_{k=1}^{p} w\left(t_{k}\right)=1, \\
\quad w\left(t_{k}\right) \in[0,1], k=1,2, \ldots, p,
\end{array}
$$

solved via Lingo software, from which time-sequence weight vector $w\left(t_{k}\right)=\left(w\left(t_{1}\right), w\left(t_{2}\right), \ldots, w\left(t_{p}\right)\right)^{T}$ is acquired.

Upon determining the time-sequence weights, the decision-making information matrix $D_{X\left(t_{k}\right)}=N\left(\mu_{i j}\left(t_{k}\right)\right.$, $\left.\sigma_{i j}\left(t_{k}\right)^{2}\right)_{m \times n}$ of different time-sequences is assembled with the DNDNWAA ${ }_{w(t)}$ operator from Formula (4) to form a comprehensive decision-making information matrix $D_{X}=N\left(\mu_{i j}, \sigma_{i j}{ }^{2}\right)_{m \times n}$ that consists of attribute and solution dimensions. The normally distributed stochastic variable that corresponds to attribute $C_{j}$, subject to solution $S_{i}$, is $X_{i j} \sim$ $N\left(\mu_{i j}, \sigma_{i j}{ }^{2}\right)$. Its corresponding normal distribution number is $x_{i j}=N\left(\mu_{i j}, \sigma_{i j}\right)$, where $\mu_{i j}=\sum_{k=1}^{p} w\left(t_{k}\right) \mu_{i j}\left(t_{k}\right)$, $\sigma_{i j}=\sqrt{\sum_{k=1}^{p} w\left(t_{k}\right)^{2} \sigma_{i j}\left(t_{k}\right)^{2}}$.

3.3. Determination of Attribute Weight. Attribute weight determination in normally distributed stochastic decisionmaking seldom stems from the characteristics of the attribute itself, and, from the characteristics of normally distributed stochastic variables, the superiority or inferiority of a variable attribute is closely related to its variance size-the smaller the variance, the better the attribute [36]. With the previous analysis, the variance characteristics of normally distributed stochastic variables can be used to determine attribute weights. Hence, this study obtains attribute weight by establishing an attribute-weight-solving optimised model based on stochastic variable variance characteristics guided by the shortest vertical projection distance.

Vertical projection distance refers to the distance between the planes that take the connections crossing the positive and negative ideal solution points as a normal vector [37]. Vertical projection distance can prevent a reversed order solution, which may occur in traditional Euclidean distance methods, and allows the characteristics of stochastic variable variances to be highlighted when calculating attribute weight in stochastic decision-making problems. Vertical projection distance has been elaborated upon in the literature [37]; in this study, it mainly provides an optimised model for determining attribute weight.

Like the comprehensive decision-making information matrix $D_{X}=N\left(\mu_{i j}, \sigma_{i j}{ }^{2}\right)_{m \times n}$, assembled based on time-seq- uences, owing to the difference in data dimensionality of different attributes, nondimensionalisation is carried out for comprehensive decision-making information, to avoid the influence of dimensionality differences on the decisionmaking result. Considering the normal distribution number $x_{i j}=N\left(\mu_{i j}, \sigma_{i j}\right)$ to which each attribute corresponds, the nondimensionalisation formulas for benefit-oriented and cost-oriented indexes and for index variance are, respectively $[11,12]$,

$$
\begin{aligned}
\tilde{\mu}_{i j} & =\frac{\mu_{i j}}{\max _{i}\left(\mu_{i j}\right)}, \\
\tilde{\mu}_{i j}\left(t_{k}\right) & =\frac{\min _{i}\left(\mu_{i j}\right)}{\mu_{i j}}, \\
\tilde{\sigma}_{i j} & =\frac{\sigma_{i j}}{\max _{i}\left(\mu_{i j}\right)} .
\end{aligned}
$$

A normal distribution number after nondimensionalisation is denoted by $\widetilde{x}_{i j}=N\left(\widetilde{\mu}_{i j}, \widetilde{\sigma}_{i j}\right)$ and the decision-making information matrix is denoted by $\widetilde{D}_{X}=N\left(\widetilde{\mu}_{i j}, \widetilde{\sigma}_{i j}{ }^{2}\right)_{m \times n}$.

Attribute weights are determined after conducting nondimensionalisation of the comprehensive decision-making information using vertical projection distance. The specific steps of this calculation are as follows.

Suppose attribute-weight vector $w=\left(w_{1}, w_{2}, \ldots, w_{n}\right)$ and denote the root extraction (i.e., standard deviation) matrix of the standardised stochastic variable based on attribute weighting as

$$
R=\left(r_{i j}\right)_{m \times n}=\left(\sqrt{\widetilde{\sigma}_{i j}^{2}}\right)_{m \times n} \times\left(w_{j}\right)_{n \times 1} .
$$

Shift the origin coordinates of matrix $R$ to the ideal variance point to obtain ideal variance $S^{+}=\left(S_{j}^{+} \mid j=\right.$ $1,2, \ldots, n)$. The shifted matrix is denoted by $Z=\left(z_{i j}\right)_{m \times n}$. 
Per the characteristics of stochastic variable variances, $S_{j}^{+}=$ $\min _{1 \leq i \leq m}\left\{\widetilde{\sigma}_{i j}{ }^{2}\right\}$ can be obtained; then

$$
\begin{aligned}
z_{i j}=\sqrt{\widetilde{\sigma}_{i j}^{2}}-S_{j}^{+}=\left(\sqrt{\widetilde{\sigma}_{i j}^{2}}-\sqrt{\widetilde{\sigma}_{j}^{+2}}\right) w_{j}, \\
\quad i=1,2, \ldots, m ; j=1,2, \ldots, n .
\end{aligned}
$$

To determine the negative ideal variance of matrix $Z$ as $H^{-}=\left(H_{j}^{-} \mid j=1,2, \ldots, m\right)$, the ideal variance is $(0,0, \ldots, 0)$, where

$$
H_{j}^{-}=z_{\eta j}=\sqrt{\widetilde{\sigma}_{i j}^{2}} w_{j}
$$

Set $\left|z_{\eta j}\right| \geq\left|z_{i j}\right|$ or $\left|\sqrt{\widetilde{\sigma}_{\eta j}^{2}}\right| \geq\left|\sqrt{\widetilde{\sigma}_{i j}^{2}}-\sqrt{\widetilde{\sigma}_{j}^{+2}}\right|$, where $1 \leq \eta \leq m$.

Vertical projection distance $V_{i}$ of each variance and the ideal variance, because the distance between the positive and negative ideal variances is constant for each solution, may be determined by

$$
V_{i}=\sum_{j=1}^{n} H_{j}^{-} \times z_{i j} .
$$

The variances in normally distributed stochastic variables $V_{i}$ can be thought of as the degree of closeness between the variance and ideal variance of all attributes in different solutions-smaller values of $V_{i}$ indicate that the variance and ideal variance of attributes in a solution are closer. To achieve more optimised stochastic variables, variances should be as small as possible. Therefore, these problems can be inverted to the following optimised ones:

$$
\begin{array}{ll}
\min & \sum_{i=1}^{m} V_{i}=\sum_{i=1}^{m} \sum_{j=1}^{n} H_{j}^{-} \times z_{i j} \\
& =\sum_{i=1}^{m} \sum_{j=1}^{n} \sqrt{\widetilde{\sigma}_{\eta j}^{2}}\left(\sqrt{\left.{\widetilde{\sigma}_{i j}^{2}}^{2}-\sqrt{\widetilde{\sigma}_{j}^{+2}}\right) w_{j}^{2}}\right. \\
\text { s.t. } & \sum_{j=1}^{n} w_{j}=1, \\
& w_{j}>0, j=1,2, \ldots, n .
\end{array}
$$

Attribute weight $w=\left(w_{1}, w_{2}, \ldots, w_{n}\right)^{T}$ can be solved via Lingo software.

3.4. Decision-Making Based on Order Relation. Upon obtaining attribute weight, comprehensive decision-making information is assembled after nondimensionalisation from attribute dimensions, per the $\mathrm{NDNWAA}_{w}$ operator in Formula (3), forming a decision-making information matrix $\widetilde{D}_{X_{i}}=N\left(\widetilde{\mu}_{i}, \widetilde{\sigma}_{i}^{2}\right)_{m \times 1}$ constituted by a single solution dimension. Because of the characteristics of expectation and variance attributes in normally distributed stochastic variables, the greater expectation $\widetilde{\mu}_{i}$ is, the smaller $\widetilde{\sigma}_{i}^{2}$ is and the greater corresponding stochastic variable $X_{i}$ is [36]. Normally, $\widetilde{\sigma}_{i}^{2}>$ 0 ; if $\widetilde{\sigma}_{i}^{2}=0$, then the normally distributed stochastic variable is degraded into a real number. Therefore, the order relation criteria between any two arbitrary normally distributed stochastic variables $X_{1} \sim N\left(\tilde{\mu}_{1}, \widetilde{\sigma}_{1}{ }^{2}\right)$ and $X_{2} \sim N\left(\tilde{\mu}_{2}, \widetilde{\sigma}_{2}{ }^{2}\right)$ can be defined as follows:

$$
\text { If } \tilde{\mu}_{1} / \widetilde{\sigma}_{1}{ }^{1}>\tilde{\mu}_{2} / \widetilde{\sigma}_{2}^{2} \text {, then } X_{1}>X_{2} \text {. }
$$

The order relation criteria of normally distributed stochastic variables state that the superiority-inferiority degree value $\left(X_{i}=X_{1}, X_{2}, \ldots, X_{m}\right)$ of related normally distributed stochastic variables can be obtained to determine the priority sequence of the solution; that is, the greater $X_{i}$ is, the more optimised corresponding ith solution is.

3.5. Summary of the New Dynamic Stochastic Decision-Making Method. In brief, the calculation steps for dynamic stochastic multiattribute decision-making that considers stochastic variable characteristics under time-sequence contingencies are as follows.

Step 1. Obtain $p$ moments of time-sequence weight vector $w\left(t_{k}\right)=\left(w\left(t_{1}\right), w\left(t_{2}\right), \ldots, w\left(t_{p}\right)\right)^{T}$ using Formula (8) after obtaining $p$ moments of dynamic stochastic decision-making information matrix $D_{X\left(t_{k}\right)}=N\left(\mu_{i j}\left(t_{k}\right), \sigma_{i j}\left(t_{k}\right)^{2}\right)_{m \times n}$.

Step 2. Assemble $p$ moments of dynamic stochastic decisionmaking information with the time-sequence weights and Formula (4) to form a comprehensive decision-making information matrix $D_{X}=N\left(\mu_{i j}, \sigma_{i j}{ }^{2}\right)_{m \times n}$ comprising attributes and solutions.

Step 3. Conduct nondimensionalisation for comprehensive decision-making information $D_{X}$, using Formulas (9) through (11) to form a decision-making information matrix $\widetilde{D}_{X}=N\left(\widetilde{\mu}_{i j}, \widetilde{\sigma}_{i j}{ }^{2}\right)_{m \times n}$ after nondimensionalisation, and obtain attribute-weight vector $w=\left(w_{1}, w_{2}, \ldots, w_{n}\right)^{T}$ as in Formula (16).

Step 4. Further assemble decision-making information matrix $\widetilde{D}_{X}$ from attributes, utilising attribute weights and Formula (3) to form a comprehensive decision-making information matrix $\widetilde{D}_{X_{i}}=N\left(\widetilde{\mu}_{i}, \widetilde{\sigma}_{i}^{2}\right)_{m \times 1}$ that is only constituted of a single solution dimension.

Step 5. Finally determine the order $\left(X_{i}=X_{1}, X_{2}, \ldots, X_{m}\right)$ of each alternative solution using the order relation criteria of normally distributed stochastic variables and further determine the priority sequence of alternative solutions based on this order.

\section{Illustrative Cases of Study}

There is a major emerging technical project seeking technical partners. Five enterprises, denoted by $S_{i}=\left\{S_{1}, S_{2}, S_{3}, S_{4}, S_{5}\right\}$, are options for potential partners or solutions. The attribute parameters for evaluating each enterprise refer to the stochastic variables subject to stochastic normal distribution: the R\&D cycle of the enterprise's emerging technology $\left(C_{1}\right)$, the market share of its emerging technology products $\left(C_{2}\right)$, the 
life of its emerging technology products $\left(C_{3}\right)$, the conversion rate of its emerging technology achievements $\left(C_{4}\right)$, and the attribute set $C_{j}=\left\{C_{1}, C_{2}, C_{3}, C_{4}\right\}$, corresponding to attribute weights $w=\left(w_{1}, w_{2}, w_{3}, w_{4}\right)$. In addition, the investor of the emerging technical project has investigated related attribute information in four historical periods for the previously mentioned enterprises and synthesised historical attribute information for reference. Suppose that the time-sequence set of different historical periods is $t_{k}=\left\{t_{1}, t_{2}, t_{3}, t_{4}\right\}$.

To obtain decision-making information for each evaluation attribute that obeys or approximately obeys the law of normal distribution, we first collect sample data from completed emerging technical project developments of each enterprise as the basic data sample $\left\{Z_{i j}^{l}\left(t_{k}\right)\right\}$. Basic data were provided by each candidate enterprise for attribute set $C_{j}=\left\{C_{1}, C_{2}, C_{3}, C_{4}\right\}$ in four different periods $\left\{t_{1}, t_{2}, t_{3}, t_{4}\right\}$. Then, the collected sample data were transformed; thus the expectation $\mu_{i j}\left(t_{k}\right)$ and variance $\sigma_{i j}\left(t_{k}\right)^{2}$ of each attribute index can be calculated by the sample data in accordance with Formulas (1) and (2). Finally, the basic data samples were converted into the original evaluation attribute information data shown in Tables 1-4.

Based on the original evaluation attribute information, per Step 1, where the time degree parameter $\lambda=0.4$, the time-sequence weights of the different time-sequences were obtained:

$$
\begin{aligned}
w\left(t_{k}\right) & =\left(w\left(t_{1}\right), w\left(t_{2}\right), w\left(t_{3}\right), w\left(t_{4}\right)\right) \\
& =(0.255,0.218,0,0.527) .
\end{aligned}
$$

The four historical time-sequences were assembled as in Step 2 and synthesised into comprehensive decision-making attribute information shown in Table 5.

Nondimensionalisation was conducted on the comprehensive original evaluation attributes in Table 5 with the procedure in Step 3, as shown in Table 6.

Attribute weights were obtained as in Step 3:

$$
w=\left(w_{1}, w_{2}, w_{3}, w_{4}\right)=(0.421,0.103,0.177,0.299) .
$$

Comprehensive decision-making information was assembled from attribute information, based on Step 4, forming comprehensive normal distribution numbers constituted by target single dimensions:

$$
\begin{aligned}
& \widetilde{D}_{X_{1}}=N\left(0.88,0.033^{2}\right), \\
& \widetilde{D}_{X_{2}}=N\left(0.934,0.032^{2}\right), \\
& \widetilde{D}_{X_{3}}=N\left(0.862,0.031^{2}\right), \\
& \widetilde{D}_{X_{4}}=N\left(0.843,0.032^{2}\right), \\
& \widetilde{D}_{X_{5}}=N\left(0.997,0.034^{2}\right) .
\end{aligned}
$$

The value of each alternative cooperative enterprise was determined based on Step 5:

$$
\begin{aligned}
X & =\left(X_{1}, X_{2}, X_{3}, X_{4}, X_{5}\right) \\
& =(26.86,29.03,28.18,26.22,29.3) .
\end{aligned}
$$

The priority sequence of the five alternative enterprises, $S_{5}>S_{2}>S_{3}>S_{1}>S_{4}$, was obtained by arranging each enterprise's value from largest to smallest and the optimal solution was determined to be $S_{5}$.

Further, the decision-making solution sequence was also calculated using the possibility degree method described in the literature $[11,12]$.

First, the comprehensive normal distribution number $\widetilde{D}_{X_{i}}(i=1,2,3,4,5)$ is converted to an interval number:

$$
\begin{aligned}
& \bar{D}_{X_{1}}=[0.782,0.979], \\
& \bar{D}_{X_{2}}=[0.838,1.031], \\
& \bar{D}_{X_{3}}=[0.771,0.954], \\
& \bar{D}_{X_{4}}=[0.747,0.94], \\
& \bar{D}_{X_{5}}=[0.895,1.099] .
\end{aligned}
$$

Then, the possibility matrices of the two compared schemes are calculated:

$$
P=\left[\begin{array}{ccccc}
0.5 & 0.361 & 0.547 & 0.595 & 0.209 \\
0.639 & 0.5 & 0.691 & 0.735 & 0.343 \\
0.453 & 0.309 & 0.5 & 0.55 & 0.153 \\
0.405 & 0.265 & 0.45 & 0.5 & 0.114 \\
0.791 & 0.657 & 0.847 & 0.886 & 0.5
\end{array}\right] .
$$

Finally, the following ranking value was acquired, based on the fuzzy compensation judgment matrix in the possibility degree method:

$$
\begin{aligned}
X & =\left(X_{1}, X_{2}, X_{3}, X_{4}, X_{5}\right) \\
& =(0.286,0.32,0.273,0.261,0.359)
\end{aligned}
$$

so that the priority order obtained from the ranking value is $S_{5}>S_{2}>S_{1}>S_{3}>S_{4}$, where $S_{5}$ is the optimal investment scheme.

Obviously, the result based on the possibility degree method described in the literature [11, 12] was consistent with the result of Section 4, indicating the rationality of the method proposed in this study. Compared with the possibility degree method, the method proposed herein requires fewer nonlinear conversions during calculation and model construction, and its calculation is simple and easy to implement. In addition, compared with similar normally distributed stochastic decision-making methods, this study considers the characteristics of normally distributed stochastic variable variances while determining attribute weight; thus, the final ordering result of the method proposed in this paper is more comprehensive.

\section{Conclusions}

This paper describes a dynamic stochastic decision-making method that fully considers the characteristics of normally distributed stochastic variable variances with time-sequence 
TABLE 1: Original evaluation attribute information at $t_{1}$.

\begin{tabular}{lcccc}
\hline & $C_{1}$ & $C_{2}$ & $C_{3}$ & $C_{4}$ \\
\hline$S_{1}$ & $N\left(716,75^{2}\right)$ & $N\left(11.8,1.8^{2}\right)$ & $N\left(8.5,1.5^{2}\right)$ & $N\left(34.3,2.2^{2}\right)$ \\
$S_{2}$ & $N\left(899,89^{2}\right)$ & $N\left(9.5,1.7^{2}\right)$ & $N\left(9.3,1.8^{2}\right)$ & $N\left(47.4,4^{2}\right)$ \\
$S_{3}$ & $N\left(653,74^{2}\right)$ & $N\left(12.5,1.1^{2}\right)$ & $N\left(7.4,1.2^{2}\right)$ & $N\left(51,4.1^{2}\right)$ \\
$S_{4}$ & $N\left(816,84^{2}\right)$ & $N\left(10.9,1.9^{2}\right)$ & $N\left(10.1,2.4^{2}\right)$ & $N\left(38,3.9^{2}\right)$ \\
$S_{5}$ & $N\left(786,74^{2}\right)$ & $N\left(12,2^{2}\right)$ & $N\left(11,1.4^{2}\right)$ & $N\left(44,4.1^{2}\right)$ \\
\hline
\end{tabular}

TABLE 2: Original evaluation attribute information at $t_{2}$.

\begin{tabular}{lcccc}
\hline & $C_{1}$ & $C_{2}$ & $C_{3}$ & $C_{4}$ \\
\hline$S_{1}$ & $N\left(671,66^{2}\right)$ & $N\left(12.4,2.1^{2}\right)$ & $N\left(6.5,0.9^{2}\right)$ & $N\left(45,3.2^{2}\right)$ \\
$S_{2}$ & $N\left(790,87^{2}\right)$ & $N\left(11.5,1.9^{2}\right)$ & $N\left(7.6,1.3^{2}\right)$ & $N\left(51,5^{2}\right)$ \\
$S_{3}$ & $N\left(816,78^{2}\right)$ & $N\left(10.5,1.2^{2}\right)$ & $N\left(11.4,1.7^{2}\right)$ & $N\left(60,4.9^{2}\right)$ \\
$S_{4}$ & $N\left(718,77^{2}\right)$ & $N\left(9.9,1.2^{2}\right)$ & $N\left(8.1,1.4^{2}\right)$ & $N\left(43,3.2^{2}\right)$ \\
$S_{5}$ & $N\left(765,74^{2}\right)$ & $N\left(13,1.8^{2}\right)$ & $N\left(7.9,1.6^{2}\right)$ & $N\left(62,5.4^{2}\right)$ \\
\hline
\end{tabular}

TABLE 3: Original evaluation attribute information at $t_{3}$.

\begin{tabular}{lcccc}
\hline & $C_{1}$ & $C_{2}$ & $C_{3}$ & $C_{4}$ \\
\hline$S_{1}$ & $N\left(790,80^{2}\right)$ & $N\left(9.7,1.3^{2}\right)$ & $N\left(10.6,1.2^{2}\right)$ & $N\left(58.8,4.9^{2}\right)$ \\
$S_{2}$ & $N\left(611,61^{2}\right)$ & $N\left(10.1,1.2^{2}\right)$ & $N\left(9.8,1.7^{2}\right)$ & $N\left(60.3,4.1^{2}\right)$ \\
$S_{3}$ & $N\left(530,47^{2}\right)$ & $N\left(12.1,2.1^{2}\right)$ & $N\left(12.1,2.4^{2}\right)$ & $N\left(37.3,2.8^{2}\right)$ \\
$S_{4}$ & $N\left(799,71^{2}\right)$ & $N\left(8.9,0.8^{2}\right)$ & $N\left(11.1,2.1^{2}\right)$ & $N\left(31.3,2.8^{2}\right)$ \\
$S_{5}$ & $N\left(854,78^{2}\right)$ & $N\left(11.9,1.4^{2}\right)$ & $N\left(12,1.7^{2}\right)$ & $N\left(53,4.2^{2}\right)$ \\
\hline
\end{tabular}

TABLE 4: Original evaluation attribute information at $t_{4}$.

\begin{tabular}{lcccc}
\hline & $C_{1}$ & $C_{2}$ & $C_{3}$ & $C_{4}$ \\
\hline$S_{1}$ & $N\left(806,81^{2}\right)$ & $N\left(10.8,1^{2}\right)$ & $N\left(11.7,1.6^{2}\right)$ & $N\left(66,5.1^{2}\right)$ \\
$S_{2}$ & $N\left(744,71^{2}\right)$ & $N\left(11.1,1.1^{2}\right)$ & $N\left(12.6,1.3^{2}\right)$ & $N\left(67,5.1^{2}\right)$ \\
$S_{3}$ & $N\left(614,65^{2}\right)$ & $N\left(12.6,1.9^{2}\right)$ & $N\left(11.8,1.4^{2}\right)$ & $N\left(54,4.8^{2}\right)$ \\
$S_{4}$ & $N\left(709,69^{2}\right)$ & $N\left(9.9,1.2^{2}\right)$ & $N\left(10.6,1.7^{2}\right)$ & $N\left(56,3.7^{2}\right)$ \\
$S_{5}$ & $N\left(883,80^{2}\right)$ & $N\left(12.7,2.2^{2}\right)$ & $N\left(11.3,1.4^{2}\right)$ & $N\left(77,5.2^{2}\right)$ \\
\hline
\end{tabular}

TABLE 5: Comprehensive decision-making attribute information.

\begin{tabular}{lcccc}
\hline & $C_{1}$ & $C_{2}$ & $C_{3}$ & $C_{4}$ \\
\hline$S_{1}$ & $N\left(753.6,48.9^{2}\right)$ & $N\left(11.4,0.8^{2}\right)$ & $N\left(9.8,0.9^{2}\right)$ & $N\left(53.3,2.8^{2}\right)$ \\
$S_{2}$ & $N\left(793.6,47.7^{2}\right)$ & $N\left(10.8,0.8^{2}\right)$ & $N\left(10.7,0.9^{2}\right)$ & $N\left(58.5,3.1^{2}\right)$ \\
$S_{3}$ & $N\left(668,32.6^{2}\right)$ & $N\left(12.1,1.1^{2}\right)$ & $N\left(10.6,0.8^{2}\right)$ & $N\left(54.5,2.9^{2}\right)$ \\
$S_{4}$ & $N\left(738.3,45.4^{2}\right)$ & $N\left(10.2,0.8^{2}\right)$ & $N\left(9.9,1.1^{2}\right)$ & $N\left(48.6,2.3^{2}\right)$ \\
$S_{5}$ & $N\left(832.5,48.9^{2}\right)$ & $N\left(12.6,1.3^{2}\right)$ & $N\left(10.5,0.9^{2}\right)$ & $N\left(65.3,3.2^{2}\right)$ \\
\hline
\end{tabular}

TABLE 6: Nondimensionalised comprehensive decision-making attribute information.

\begin{tabular}{lcccc}
\hline & $C_{1}$ & $C_{2}$ & $C_{3}$ & $C_{4}$ \\
\hline$S_{1}$ & $N\left(0.905,0.059^{2}\right)$ & $N\left(0.906,0.066^{2}\right)$ & $N\left(0.914,0.089^{2}\right)$ & $N\left(0.817,0.043^{2}\right)$ \\
$S_{2}$ & $N\left(0.953,0.057^{2}\right)$ & $N\left(0.856,0.066^{2}\right)$ & $N\left(1,0.082^{2}\right)$ & $N\left(0.896,0.047^{2}\right)$ \\
$S_{3}$ & $N\left(0.802,0.051^{2}\right)$ & $N\left(0.963,0.085^{2}\right)$ & $N\left(0.993,0.083^{2}\right)$ & $N\left(0.835,0.045^{2}\right)$ \\
$S_{4}$ & $N\left(0.887,0.055^{2}\right)$ & $N\left(0.807,0.067^{2}\right)$ & $N\left(0.931,0.106^{2}\right)$ & $N\left(0.744,0.035^{2}\right)$ \\
$S_{5}$ & $N\left(1,0.059^{2}\right)$ & $N\left(1,0.105^{2}\right)$ & $N\left(0.983,0.083^{2}\right)$ & $N\left(1,0.048^{2}\right)$ \\
\hline
\end{tabular}


contingencies. It first obtains time-sequence weights by combining time-degree theory and TOPSIS. It calculates attribute weights based on the characteristics of normally distributed stochastic variable variances and vertical projection distance. Decision-making information is then assembled from the attribute and time-sequence weights via related operators, to obtain the stochastic normally distributed comprehensive decision-making matrix constituted by target single dimensions, finally providing the priority sequence of alternative solutions using order relation criteria. The primary contributions of this paper are as follows:

(1) In time-sequence weight calculations, this method fully considers decision-makers' preference to "stress the present rather than the past," which more accurately models real-world decision-making processes.

(2) The characteristics of normally distributed stochastic variable variances are highlighted in attribute-weight calculations and solution priority sequence ordering, making the final ordering result more comprehensive and reasonable.

(3) The method has a clear theoretical backing, requires only simple calculation, and is easy to implement. It provides a new, broadly applicable way of thinking of dynamic stochastic decision-making problems with time-sequence contingencies.

Our future study will extend the proposed method for multiattribute decision-making problems with intuitionistic fuzzy numbers and two-dimensional linguistic information.

\section{Additional Points}

Highlights. Stochastic decision-making is based on variance and time-sequence characteristics. A time-sequence weight calculation in line with TOPSIS and time degree is introduced. A new vertical projection distance-based attributeweight model is proposed. The final decision-making result in accordance with the order relation criteria is given.

\section{Competing Interests}

The authors declare that they have no competing interests.

\section{Acknowledgments}

This research was supported by the China Postdoctoral Science Foundation Funded Project (2016M600889), Natural Science Foundation of China (71673018), and National Fund Support Project on Social Science of China (14BGL007).

\section{References}

[1] J. Q. Wang and J. Ren, "Stochastic multi-criteria decisionmaking method based on the WC-OWA operator," Control and Decision, vol. 22, no. 12, pp. 1429-1432, 2007.

[2] C. Tan, W. H. Ip, and X. Chen, "Stochastic multiple criteria decision making with aspiration level based on prospect stochastic dominance," Knowledge-Based Systems, vol. 70, pp. 231-241, 2014.

[3] R. R. Yager and N. Alajlan, "Probability weighted means as surrogates for stochastic dominance in decision making," Knowledge-Based Systems, vol. 66, pp. 92-98, 2014.

[4] V. Prado-Lopez, T. P. Seager, M. Chester, L. Laurin, M. Bernardo, and S. Tylock, "Stochastic multi-attribute analysis (SMAA) as an interpretation method for comparative lifecycle assessment (LCA)," International Journal of Life Cycle Assessment, vol. 19, no. 2, pp. 405-416, 2014.

[5] S. Jalali, M. Seifbarghy, J. Sadeghi, and S. Ahmadi, "Optimizing a bi-objective reliable facility location problem with adapted stochastic measures using tuned-parameter multi-objective algorithms," Knowledge-Based Systems, vol. 95, pp. 45-57, 2016.

[6] G. T. Jang, "Method for hybrid stochastic multiple attribute decision making considering decision maker's psychological behavior," Chinese Journal of Management Science, vol. 22, pp. 78-84, 2014.

[7] H. Yu, P. Liu, and F. Jin, "Research on the stochastic hybrid multi-attribute decision making method based on prospect theory," Scientia Iranica, vol. 21, no. 3, pp. 1105-1119, 2014.

[8] J. Hu and L. Yang, "Dynamic stochastic multi-criteria decision making method based on cumulative prospect theory and set pair analysis," Systems Engineering Procedia, vol. 1, pp. 432-439, 2011.

[9] J. J. Hao, J. J. Zhu, and S. F. Liu, "A method for multi-stage stochastic multi-criteria decision making concerning prospect theory," Chinese Journal of Management Science, vol. 1, pp. 7381, 2015.

[10] X. Liang and Y. P. Jiang, "Method of stochastic multi-attribute decision making with 2-tuple aspirations considering regret behavior," Journal of Systems Engineering, vol. 30, pp. 19-27, 2015.

[11] X.-F. Wang and X.-J. Yang, "Dynamic stochastic multiple attribute decision making method with incomplete certain information," System Engineering Theory and Practice, vol. 30, no. 2, pp. 332-338, 2010.

[12] B. Sun and X.-F. Xu, "A dynamic stochastic decision-making method based on discrete time sequences," Knowledge-Based Systems, vol. 105, pp. 23-28, 2016.

[13] P. Liu and X. Yu, "2-dimension uncertain linguistic power generalized weighted aggregation operator and its application in multiple attribute group decision making," Knowledge-Based Systems, vol. 57, pp. 69-80, 2014.

[14] P. Liu and L. Shi, “The generalized hybrid weighted average operator based on interval neutrosophic hesitant set and its application to multiple attribute decision making," Neural Computing and Applications, vol. 26, no. 2, pp. 457-471, 2015.

[15] Z. S. Xu and R. R. Yager, "Dynamic intuitionistic fuzzy multiple attribute decision making," International Journal of Approximate Reasoning, vol. 48, pp. 246-262, 2008.

[16] C. Tan and X. Chen, "Dynamic similarity measures between intuitionistic fuzzy sets and its application," International Journal of Fuzzy Systems, vol. 16, no. 4, pp. 511-519, 2014.

[17] J. H. Park, H. J. Cho, and Y. C. Kwun, "Extension of the VIKOR method to dynamic intuitionistic fuzzy multiple attribute decision making," Computers \& Mathematics with Applications, vol. 65, no. 4, pp. 731-744, 2013.

[18] Z. Yue, “TOPSIS-based group decision-making methodology in intuitionistic fuzzy setting," Information Sciences, vol. 277, pp. 141-153, 2014. 
[19] Q. Cao, J. Wu, and C. Liang, "An intuitionsitic fuzzy judgement matrix and TOPSIS integrated multi-criteria decision making method for green supplier selection," Journal of Intelligent \& Fuzzy Systems, vol. 28, no. 1, pp. 117-126, 2015.

[20] P. Liu and Y. Liu, "An approach to multiple attribute group decision making based on intuitionistic trapezoidal fuzzy power generalized aggregation operator," International Journal of Computational Intelligence Systems, vol. 7, no. 2, pp. 291-304, 2014.

[21] P. Liu and F. Jin, "A multi-attribute group decision-making method based on weighted geometric aggregation operators of interval-valued trapezoidal fuzzy numbers," Applied Mathematical Modelling. Simulation and Computation for Engineering and Environmental Systems, vol. 36, no. 6, pp. 2498-2509, 2012.

[22] Z.-S. Chen and Y.-L. Li, "Approach for normal triangular fuzzy stochastic multiple attribute decision making based on prospect mean-variance rule," Control and Decision, vol. 29, no. 7, pp. 1239-1249, 2014.

[23] P. Liu and Y. Wang, "Multiple attribute decision-making method based on single-valued neutrosophic normalized weighted Bonferroni mean," Neural Computing and Applications, vol. 25, no. 7-8, pp. 2001-2010, 2014.

[24] Y. Li, X. Liu, and Y. Chen, "Supplier selection using axiomatic fuzzy set and TOPSIS methodology in supply chain management," Fuzzy Optimization and Decision Making. A Journal of Modeling and Computation Under Uncertainty, vol. 11, no. 2, pp. 147-176, 2012.

[25] P. D. Liu and L. L. Shi, "The generalized hybrid weighted average operator based on interval neutrosophic hesitant set and its application to multiple attribute decision making," Neural Computing and Applications, vol. 26, no. 2, pp. 457-471, 2015.

[26] Z. Xu, "On multi-period multi-attribute decision making," Knowledge-Based Systems, vol. 21, no. 2, pp. 164-171, 2008.

[27] R. Sadiq and S. Tesfamariam, "Probability density functions based weights for ordered weighted averaging (OWA) operators: an example of water quality indices," European Journal of Operational Research, vol. 182, no. 3, pp. 1350-1368, 2007.

[28] X. Cao, G. W. Liu, and X. M. Fu, "Analysis on group decision making of industry-university-research partners choice based on preferences and dynamic intuition," Operations Research Management Science, vol. 4, pp. 33-41, 2013.

[29] Y. Liu, J. Forrest, S.-F. Liu, H.-H. Zhao, and L.-R. Jian, "Dynamic multiple attribute grey incidence decision making mothed based on interval valued intuitionisitc fuzzy number," Kongzhi yu Juece/Control and Decision, vol. 28, no. 9, pp. 1303-1321, 2013.

[30] X.-Z. Zhang, C.-X. Zhu, and L. Zhu, "A method of dynamic multi-attribute decision making based on variable weight," Control and Decision, vol. 29, no. 3, pp. 494-498, 2014.

[31] X.-H. Xu and Q.-F. Wan, "Method for continuous stochastic multi-attribute large group decision making," Systems Engineering and Electronics, vol. 34, no. 10, pp. 2084-2089, 2012.

[32] V. J. Easton and J. H. McColl, Statistics Glossary v1.1, 1997.

[33] R. Lahdelma, S. Makkonen, and P. Salminen, "Multivariate Gaussian criteria in SMAA," European Journal of Operational Research, vol. 170, no. 3, pp. 957-970, 2006.

[34] R. R. Yager, "On ordered weighted averaging aggregation operators in multicriteria decision making," IEEE Transactions on Systems, Man, and Cybernetics, vol. 18, pp. 183-190, 1988.

[35] X. Z. Zhang and C. X. Zhu, "Generalized precedence ordering method with ranking preference for time series-based multiattribute decision making," Chinese Journal of Management Science, vol. 22, pp. 105-111, 2014.
[36] S. B. Graves and J. L. Ringuest, "Probabilistic dominance criteria for comparing uncertain alternatives: a tutorial," Omega, vol. 37, no. 2, pp. 346-357, 2009.

[37] X. Y. Hua and J. X. Tan, "Revised TOPSIS method based on vertical projection distance-vertical projection method," Systems Engineering-Theory \& Practice, vol. 1, pp. 114-119, 2004. 


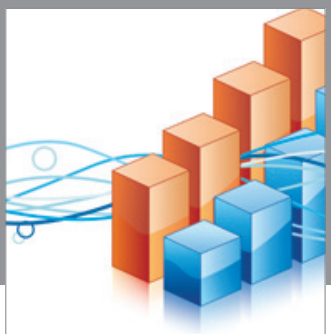

Advances in

Operations Research

vatem alat4

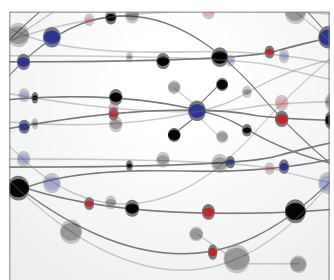

\section{The Scientific} World Journal
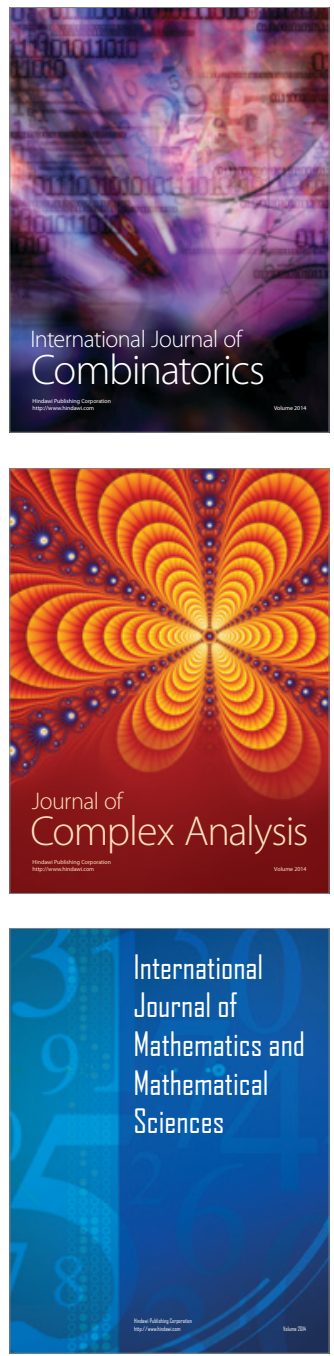
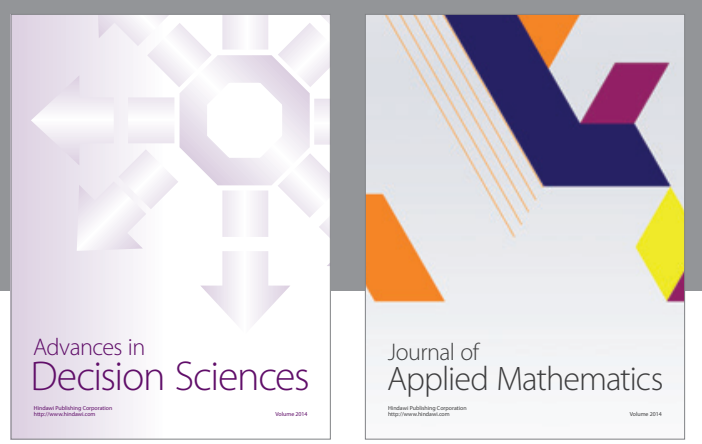

Algebra

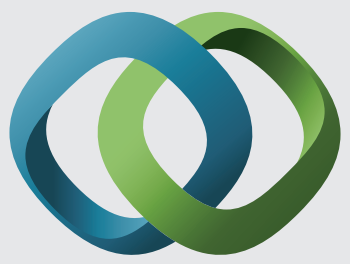

\section{Hindawi}

Submit your manuscripts at

https://www.hindawi.com
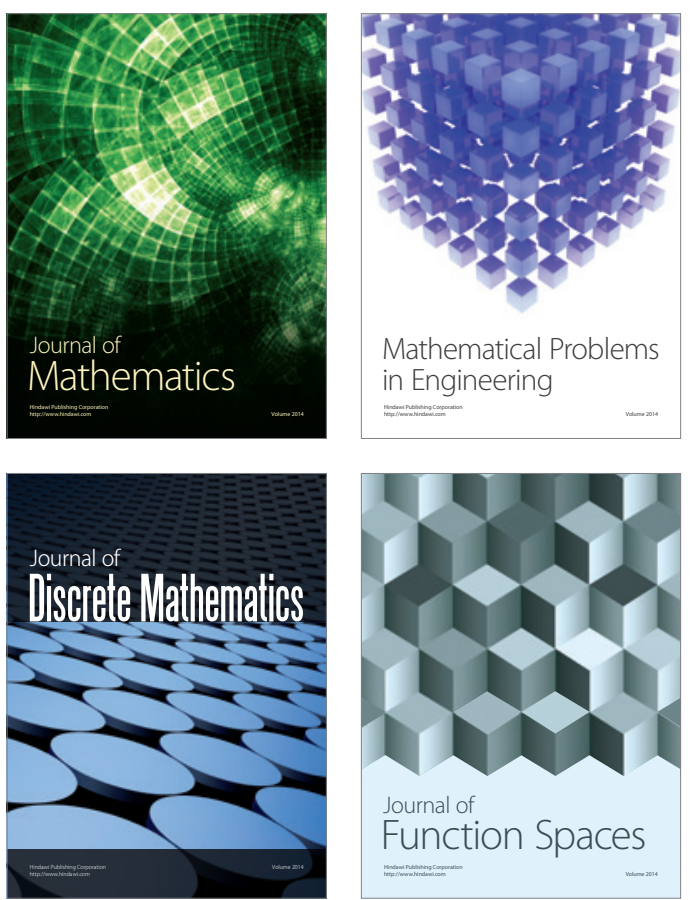

Mathematical Problems in Engineering
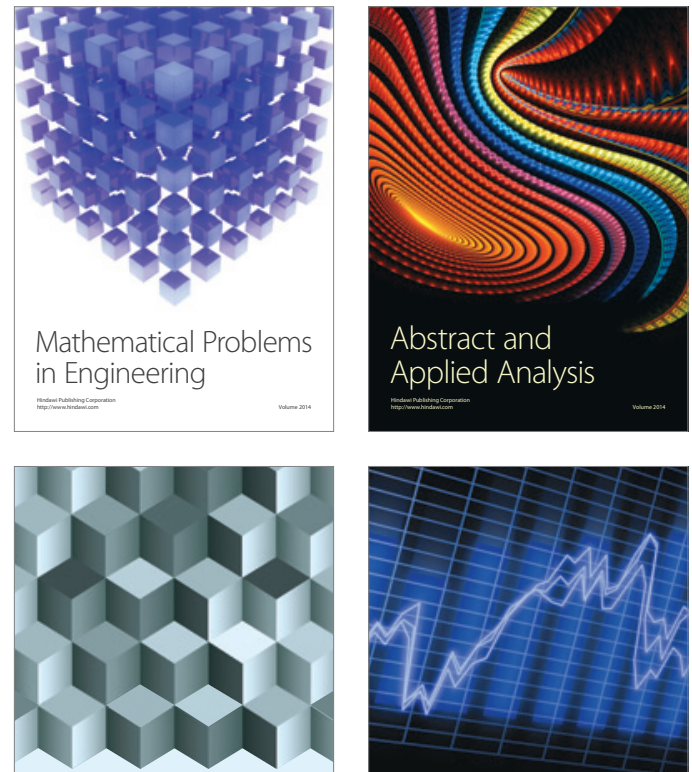

Journal of

Function Spaces

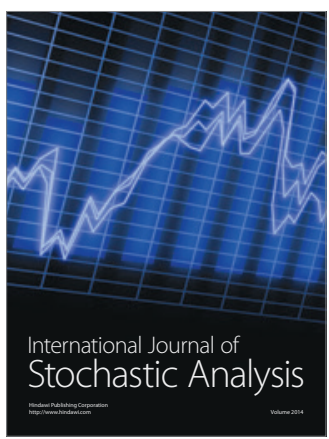

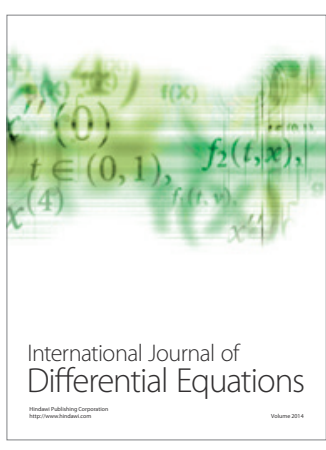
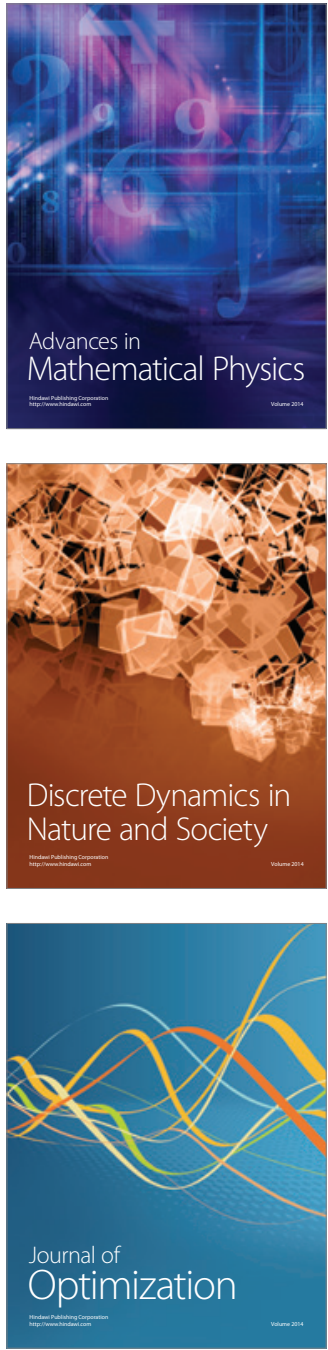